\title{
CONVOLUTIONS OF GENERIC ORBITAL MEASURES IN COMPACT SYMMETRIC SPACES
}

\author{
SANJIV KUMAR GUPTA and KATHRYN E. HARE ${ }^{凶}$
}

(Received 29 September 2008)

\begin{abstract}
We prove that in any compact symmetric space, $G / K$, there is a dense set of $a_{1}, a_{2} \in G$ such that if $\mu_{j}=m_{K} * \delta_{a_{j}} * m_{k}$ is the $K$-bi-invariant measure supported on $K a_{j} K$, then $\mu_{1} * \mu_{2}$ is absolutely continuous with respect to Haar measure on $G$. Moreover, the product of double cosets, $K a_{1} K a_{2} K$, has nonempty interior in $G$.
\end{abstract}

2000 Mathematics subject classification: primary 43A80; secondary 58C35, 53C35.

Keywords and phrases: compact symmetric space, double coset, absolutely continuous measure.

\section{Introduction}

In a now classical paper [4], Dunkl proved that the convolution of the surface measure of a sphere in $\mathbb{R}^{n}$ with itself is absolutely continuous with respect to Lebesgue measure in $\mathbb{R}^{n}$. Motivated by this result, Ragozin [9] considered the analogous problem in the setting of a compact, symmetric space $G / K$ and showed that if $\mu_{j}$ are $K$-bi-invariant, continuous measures, then $\mu_{1} * \cdots * \mu_{\operatorname{dim} G / K}$ is absolutely continuous with respect to the Haar measure on $G$. In particular, this is true when $\mu_{j}$ are the $K$-orbital surface measures supported on the double cosets $K a_{j} K$, with $a_{j}$ not in the normalizer of $K$ in $G$. These singular measures are given by

$$
\mu_{j}=\mu_{a_{j}}=m_{K} * \delta_{a_{j}} * m_{K}
$$

where $m_{K}$ denotes the Haar measure on $K$. Equivalently, the $\operatorname{dim} G / K$-fold product of the double cosets $K a_{j} K$ has nonempty interior for all such $a_{j}$.

Recently, the authors [5] proved that for the special case of the symmetric space $S U(n) / S O(n)$ the number of convolution powers (or double cosets in the product) could be reduced from the dimension of the symmetric space to the rank +1 , and that this is sharp for particular $a_{j} \in S U(n)$.

In this paper, we prove that for any compact symmetric space there is a dense subset $D \subseteq G$ such that if $a_{1}, a_{2} \in D$, then $\mu_{a_{1}} * \mu_{a_{2}}$ is absolutely continuous with respect to

This research was supported in part by NSERC and the Sultan Qaboos University.

(C) 2009 Australian Mathematical Society 0004-9727/2009 \$16.00 
the Haar measure on $G$ and the product of double cosets, $K a_{1} K a_{2} K$, has nonempty interior. General results of Ricci and Stein [10] then imply that the convolution product actually belongs to $L^{p}(G)$ for some $p>1$.

One example of this is when $H$ is a compact, simple, connected Lie group, $G=H \times H$ and $K=\{(h, h) \mid h \in H\}$. Then $G / K$ is homeomorphic to $H$, double cosets correspond to conjugacy classes, and the $K$-bi-inviariant measures on $G / K$ can be identified with the central measures on $H$. Using the representation theory of compact Lie groups, a stronger result has been proved in this case; namely, $\mu_{a_{1}} * \mu_{a_{2}} \in L^{2}(H)$ for a dense set of elements of $H$ [6]. It would be interesting to know whether this stronger result holds for general compact symmetric spaces as well. This may require further development of the $L^{2}$ theory for symmetric spaces (see $[2,8])$. Smoothness properties of these orbital measures were also investigated in $[3,11]$.

\section{Notation and basic facts}

2.1. Restricted roots and root vectors Let $G$ be a compact, connected, semi-simple Lie group and suppose $\theta$ is a Cartan involution that fixes the closed Lie subgroup $K$. The quotient space $G / K$ is known as a compact symmetric space. We denote by $N_{G}(K)$ the normalizer of $K$ in $G$.

We shall write $\mathfrak{g}=\mathfrak{k} \oplus \mathfrak{p}$ for the corresponding Cartan decomposition of the Lie algebra $\mathfrak{g}$ of $G .^{1}$ Thus $\mathfrak{p}$ is the -1 eigenspace of $\theta$. Let $\mathfrak{a} \subseteq \mathfrak{p}$ be a maximal abelian subspace and extend this to a maximal abelian subalgebra, $t$, of the Lie algebra $\mathfrak{g}$. We write $\mathfrak{m}$ for the subspace of $\mathfrak{k}$ that commutes with $\mathfrak{a}$. For a classification of compact symmetric spaces we refer the reader to [1, p. 219] or [7, p. 518].

Let $\tau$ be the conjugation of $\mathfrak{g}$ which gives the complexified Lie algebra $\mathfrak{g}^{\mathbb{C}}$ and extend $\theta$ by linearity to $\mathfrak{g}^{\mathbb{C}}$. More generally $\mathfrak{a}^{\mathbb{C}}, t^{\mathbb{C}}$, and so on, will denote the complexification of the corresponding subspace.

Suppose $\Phi$ is the set of roots of $\mathfrak{g}^{\mathbb{C}}$ with respect to $t^{\mathbb{C}}$. We consider the roots which do not vanish identically on $\mathfrak{a}^{\mathbb{C}}$ and let $\Sigma\left(\Sigma^{+}\right)$denote the corresponding set of (positive) restricted roots. We denote by $g_{\alpha}^{R}$ the restricted root space for the restricted $\operatorname{root} \alpha \in \Sigma$ :

$$
g_{\alpha}^{R}=\left\{X \in \mathfrak{g}^{\mathbb{C}} \mid[H, X]=i \alpha(H) X \text { for all } H \in \mathfrak{a}\right\} .
$$

The restricted root vectors are the nonzero vectors in $g_{\alpha}^{R}$. Similarly, $g_{\alpha}$ will denote the root space of the root $\alpha \in \Phi$.

In contrast to the situation for root spaces, restricted root spaces need not be onedimensional. Indeed,

$$
g_{\alpha}^{R}=\sum g_{\beta}
$$

where the sum is over all root vectors $\beta$ such that $\left.\beta\right|_{\mathfrak{a}}=\alpha$. The complexified Lie algebra can be decomposed as

$$
\mathfrak{g}^{\mathbb{C}}=t^{\mathbb{C}} \oplus \sum_{\alpha \in \Phi} g_{\alpha}=\mathfrak{a}^{\mathbb{C}} \oplus \mathfrak{m}^{\mathbb{C}} \oplus \sum_{\alpha \in \Sigma} g_{\alpha}^{R} .
$$

\footnotetext{
${ }^{1}$ Following Ragozin, we define our Lie algebras as right-invariant vector fields.
} 
If $H \in \mathfrak{a}$, then $\theta(H)=-H$ and $\tau(H)=H$. Thus if $X_{\alpha} \in g_{\alpha}^{R}$, then

$$
\left[H, \theta\left(X_{\alpha}\right)\right]=\theta\left[\theta(H), X_{\alpha}\right]=-\theta\left[H, X_{\alpha}\right]=-i \alpha(H) \theta\left(X_{\alpha}\right)
$$

and

$$
\left[H, \tau\left(X_{\alpha}\right)\right]=\tau\left[\tau(H), X_{\alpha}\right]=\tau\left[H, X_{\alpha}\right]=-i \alpha(H) \tau\left(X_{\alpha}\right),
$$

with the final inequality coming because $\tau$ is conjugate linear. Hence both $\theta$ and $\tau$ map $g_{\alpha}^{R}$ to $g_{-\alpha}^{R}$.

2.2. Regular elements Given a restricted root $\alpha \in \Sigma$ and $a \in \exp \mathfrak{a}$, say $a=\exp (A)$ for $A \in \mathfrak{a}$, we set $\alpha(a)=\alpha(A)$. We call the element $a \in \exp \mathfrak{a}$ regular if $\alpha(a) \neq 0$ $\bmod \pi$ for any $\alpha \in \Sigma$.

It follows from the Cartan decomposition that the double cosets, $K a K$, can be indexed by the elements in $\exp \mathfrak{a} \subseteq G$. The regular elements in $\exp \mathfrak{a}$ are dense in $\exp \mathfrak{a}$, and the elements $g \in G$ with $K g K=K a K$ for some regular $a \in \exp \mathfrak{a}$ are dense in $G$. We shall show in Corollary 2.3 that if $a$ is regular, then $a \notin N_{G}(K)$.

2.3. Preliminary results For $E_{\alpha} \in g_{\alpha}^{R}$ set

$$
\begin{aligned}
F_{\alpha} & =E_{\alpha}+\tau E_{\alpha}+\theta\left(E_{\alpha}+\tau E_{\alpha}\right), \\
F_{\alpha}^{\prime} & =i\left(E_{\alpha}-\tau E_{\alpha}+\theta\left(E_{\alpha}-\tau E_{\alpha}\right)\right), \\
G_{\alpha} & =E_{\alpha}+\tau E_{\alpha}-\theta\left(E_{\alpha}+\tau E_{\alpha}\right), \\
G_{\alpha}^{\prime} & =i\left(E_{\alpha}-\tau E_{\alpha}-\theta\left(E_{\alpha}-\tau E_{\alpha}\right)\right) .
\end{aligned}
$$

Of course, $F_{\alpha}, F_{\alpha}^{\prime}, G_{\alpha}, G_{\alpha}^{\prime} \in g_{\alpha}^{R} \oplus g_{-\alpha}^{R}$. All four vectors are fixed by $\tau$ and hence belong to $\mathfrak{g}$. Moreover, $F_{\alpha}, F_{\alpha}^{\prime}$ are fixed by $\theta$ and thus belong to $\mathfrak{k}$, while $G_{\alpha}, G_{\alpha}^{\prime}$ are negated by $\theta$ and hence are in $\mathfrak{p}$. If $E_{\alpha}^{(1)}, \ldots, E_{\alpha}^{\left(m_{\alpha}\right)}$ is a basis for $g_{\alpha}^{R}$ and $F_{\alpha}^{(j)}, F_{\alpha}^{(j) \prime}$, $G_{\alpha}^{(j)}, G_{\alpha}^{(j) \prime}, j=1, \ldots, m_{\alpha}$ are the corresponding vectors, then

$$
\mathfrak{k}=\operatorname{span}\left\{F_{\alpha}^{(j)}, F_{\alpha}^{(j) \prime} \mid j=1, \ldots, m_{\alpha} ; \alpha \in \Sigma\right\} \oplus \mathfrak{m}
$$

and

$$
\mathfrak{p}=\operatorname{span}\left\{G_{\alpha}^{(j)}, G_{\alpha}^{(j) \prime} \mid j=1, \ldots, m_{\alpha} ; \alpha \in \Sigma\right\} \oplus \mathfrak{a} .
$$

We shall follow the usual practice of writing $\operatorname{Ad}(a)$ for the action of the group on the Lie algebra. For $E_{\alpha} \in g_{\alpha}^{R}$, we have $\operatorname{Ad}(a) E_{\alpha}=e^{i \alpha(a)} E_{\alpha}$, thus

$$
\begin{gathered}
\operatorname{Ad}(a) \theta E_{\alpha}=e^{-i \alpha(a)} \theta E_{\alpha}, \\
\operatorname{Ad}(a) \tau E_{\alpha}=e^{-i \alpha(a)} \tau E_{\alpha} .
\end{gathered}
$$

Simple calculation shows that this implies the following result.

LEMMA 2.1.

(i) $\operatorname{Ad}(a) F_{\alpha}=\cos \alpha(a) F_{\alpha}+\sin \alpha(a) G_{\alpha}^{\prime}$.

(ii) $\operatorname{Ad}(a) F_{\alpha}^{\prime}=\cos \alpha(a) F_{\alpha}^{\prime}-\sin \alpha(a) G_{\alpha}$. 
COROLlaRY 2.2. If $a$ is regular, then $\operatorname{Ad}(a) \mathfrak{k}+\mathfrak{k}=\mathfrak{g} \ominus \mathfrak{a}$.

PROOF. Since $a$ is regular, $\sin \alpha(a) \neq 0$ for any $\alpha \in \Sigma$ and thus

$$
\operatorname{span}\left\{F_{\alpha}, F_{\alpha}^{\prime}, \operatorname{Ad}(a) F_{\alpha}, \operatorname{Ad}(a) F_{\alpha}^{\prime}\right\}=\operatorname{span}\left\{F_{\alpha}, F_{\alpha}^{\prime}, G_{\alpha}, G_{\alpha}^{\prime}\right\} .
$$

Since $a \in N_{G}(K)$ if and only if $\operatorname{Ad}(a) \mathfrak{k} \subseteq \mathfrak{k}$, similar reasoning shows the following result.

COROLlary 2.3. An element a belongs to the $N_{G}(K)$ if and only if $\alpha(a)=0 \bmod \pi$ for all $\alpha \in \Sigma$.

There is a particular restricted root vector that we shall be interested in.

LEMMA 2.4. For each restricted root $\alpha$, there is a restricted root vector $E_{\alpha} \in g_{\alpha}^{R}$ such that $\left[E_{\alpha}, \theta\left(E_{\alpha}\right)\right] \in i \mathfrak{a}$.

PRoOF. Let $\tilde{\mathfrak{g}}=\mathfrak{k}+i \mathfrak{p}$. By [1, Proposition 32.5] there is a choice $E_{\alpha} \in \tilde{\mathfrak{g}} \cap g_{\alpha}^{R}$ with $\theta\left(E_{\alpha}\right) \in \tilde{\mathfrak{g}} \cap g_{-\alpha}^{R}$. Hence $\left[E_{\alpha}, \theta\left(E_{\alpha}\right)\right] \in \tilde{\mathfrak{g}}$.

Note that $\theta[X, \theta(X)]=-[X, \theta(X)]$, so $[X, \theta(X)] \in \mathfrak{p}^{\mathbb{C}}$. An application of the Jacobi identity proves that for any $H \in \mathfrak{a}^{\mathbb{C}}$ and $X \in g_{\alpha}^{R}$,

$$
\begin{aligned}
{[H,[X, \theta(X)]] } & =-[X,[\theta(X), H]]-[\theta(X),[H, X]] \\
& =-[X, i \alpha(H) \theta(X)]-[\theta(X), i \alpha(H) X]=0 .
\end{aligned}
$$

Hence $[X, \theta(X)]$ commutes with all $H \in \mathfrak{a}^{\mathbb{C}}$. Since $\mathfrak{a}^{\mathbb{C}}$ is a maximal abelian subspace of $\mathfrak{p}^{\mathbb{C}}$, it follows that $[X, \theta(X)] \in \mathfrak{a}^{\mathbb{C}}$.

Consequently, $\left[E_{\alpha}, \theta\left(E_{\alpha}\right)\right] \in \widetilde{\mathfrak{g}} \cap \mathfrak{a}^{\mathbb{C}}=i \mathfrak{a}$.

Let $\mathcal{P}: \mathfrak{g} \rightarrow \mathfrak{a}$ denote the projection map. Here are some other elementary facts that will be of use to us later.

LEMMA 2.5 .

(i) $\left[F_{\alpha}, G_{\alpha}^{\prime}\right]-\left[F_{\alpha}^{\prime}, G_{\alpha}\right]=-4(I-\theta) i\left[E_{\alpha}, \tau\left(E_{\alpha}\right)\right]=-8 \mathcal{P}\left(i\left[E_{\alpha}, \tau E_{\alpha}\right]\right)$.

(ii) If $E_{\alpha}$ is chosen with $\left[E_{\alpha}, \theta\left(E_{\alpha}\right)\right] \in i \mathfrak{a}$, then $\left[F_{\alpha}, G_{\alpha}\right]=\left[F_{\alpha}^{\prime}, G_{\alpha}^{\prime}\right]$.

PROOF. The first equality in (i) is a straightforward computation. Because $\tau\left(E_{\alpha}\right) \in$ $g_{-\alpha}^{R}$, then $i\left[E_{\alpha}, \tau E_{\alpha}\right] \in \mathfrak{m}^{\mathbb{C}} \oplus \mathfrak{a}^{\mathbb{C}}$. But also $\tau\left(i\left[E_{\alpha}, \tau E_{\alpha}\right]\right)=-i\left[\tau E_{\alpha}, E_{\alpha}\right]=$ $i\left[E_{\alpha}, \tau E_{\alpha}\right]$ and therefore $i\left[E_{\alpha}, \tau E_{\alpha}\right] \in \mathfrak{g}$. Since $(I-\theta) / 2$ projects from $\mathfrak{g}$ onto $\mathfrak{p}$, we obtain the second equality.

For (ii) one can first check that, for any root $\alpha$,

$$
\begin{aligned}
& {\left[F_{\alpha}, G_{\alpha}\right]-\left[F_{\alpha}^{\prime}, G_{\alpha}^{\prime}\right]} \\
& \quad=2\left[\theta\left(E_{\alpha}+\tau\left(E_{\alpha}\right)\right), E_{\alpha}+\tau\left(E_{\alpha}\right)\right]+2\left[\theta\left(E_{\alpha}-\tau\left(E_{\alpha}\right)\right), E_{\alpha}-\tau\left(E_{\alpha}\right)\right] \\
& \quad=4\left(\left[\theta\left(E_{\alpha}\right), E_{\alpha}\right]+\tau\left[\theta\left(E_{\alpha}\right), E_{\alpha}\right]\right),
\end{aligned}
$$

with the latter equality due to the fact that $\theta \tau=\tau \theta$. But $\left[\theta\left(E_{\alpha}\right), E_{\alpha}\right] \in i \mathfrak{a}$, so $\tau\left[\theta\left(E_{\alpha}\right), E_{\alpha}\right]=-\left[\theta\left(E_{\alpha}\right), E_{\alpha}\right]$. 
We shall also make use of the following technical result which we could not find in the literature. We recall that $\mathfrak{g}^{\mathbb{C}}$ admits a Weyl basis $\left\{X_{\beta} \mid \beta \in \Phi^{+}\right\}$where $X_{\beta} \in g_{\beta}$ [7, p. 421]. Such a basis has the property that $\tau\left(X_{\beta}\right)=-X_{-\beta}$ and $\left[X_{\beta}, X_{-\beta}\right]=H_{\beta}$ where $H_{\beta}$ is the linear functional on $\mathfrak{t}^{\mathbb{C}}$ given by $H_{\beta}(t)=\beta(t)$.

LEMMA 2.6. For any nonzero $E_{\alpha} \in g_{\alpha}^{R}, \mathcal{P}\left(i\left[E_{\alpha}, \tau E_{\alpha}\right]\right)=\left.c_{\alpha} H_{\alpha}\right|_{\mathfrak{a}}$ where $c_{\alpha}$ is a nonzero constant (depending on $E_{\alpha}$ ).

PROOF. Since $g_{\alpha}^{R}=\sum_{\left.\beta\right|_{\mathfrak{a}}=\alpha} g_{\beta}$, we can write

$$
E_{\alpha}=\sum_{\left.\beta\right|_{\mathfrak{a}}=\alpha} b_{\beta} X_{\beta},
$$

where $\left\{X_{\beta} \mid \beta \in \Phi\right\}$ is a Weyl basis of $\mathfrak{g}^{\mathbb{C}}$. Thus

$$
\begin{aligned}
{\left[E_{\alpha}, \tau E_{\alpha}\right] } & =\left[\sum_{\left.\beta\right|_{\mathfrak{a}}=\alpha} b_{\beta} X_{\beta}, \tau\left(\sum_{\left.\beta\right|_{\mathfrak{a}}=\alpha} b_{\beta} X_{\beta}\right)\right] \\
& =\left[\sum_{\left.\beta\right|_{\mathfrak{a}}=\alpha} b_{\beta} X_{\beta},-\sum_{\left.\beta\right|_{\mathfrak{a}}=\alpha} \overline{b_{\beta}} X_{-\beta}\right] \\
& =-\sum_{\beta,\left.\gamma\right|_{\mathfrak{a}}=\alpha} b_{\beta} \overline{b_{\gamma}}\left[X_{\beta}, X_{-\gamma}\right] .
\end{aligned}
$$

Consequently,

$$
\mathcal{P}\left(i\left[E_{\alpha}, \tau E_{\alpha}\right]\right)=-\mathcal{P}\left(\sum_{\left.\beta\right|_{\mathfrak{a}}=\alpha} i\left|b_{\beta}\right|^{2}\left[X_{\beta}, X_{-\beta}\right]\right)-\mathcal{P}\left(\sum_{\beta \neq \gamma} i b_{\beta} \overline{b_{\gamma}}\left[X_{\beta}, X_{-\gamma}\right]\right) .
$$

When $\beta \neq \gamma$, then $\left[X_{\beta}, X_{-\gamma}\right]$ either belongs to the root space $g_{\beta-\gamma}$ (if $\beta-\gamma$ is a root) or is zero. In either case, the projection onto $\mathfrak{a}$ is zero. Hence

$$
\mathcal{P}\left(i\left[E_{\alpha}, \tau E_{\alpha}\right]\right)=-\left.\sum_{\left.\beta\right|_{\mathfrak{a}}=\alpha} i\left|b_{\beta}\right|^{2} H_{\beta}\right|_{\mathfrak{a}}
$$

Since $\left.\beta\right|_{\mathfrak{a}}=\alpha,\left.H_{\beta}\right|_{\mathfrak{a}}=\left.H_{\alpha}\right|_{\mathfrak{a}}$. Thus $\mathcal{P}\left(i\left[E_{\alpha}, \tau E_{\alpha}\right]\right)=\left.c_{\alpha} H_{\alpha}\right|_{\mathfrak{a}}$ where

$$
c_{\alpha}=-i \sum_{\left.\beta\right|_{\mathfrak{a}}=\alpha}\left|b_{\beta}\right|^{2} \neq 0
$$

as $E_{\alpha} \neq 0$.

\section{Main theorem}

By a measure we mean a finite regular Borel measure on $G$. The measure $\mu$ is $K$ bi-invariant if $\mu\left(k_{1} S k_{2}\right)=\mu(S)$ for all $k_{1}, k_{2} \in K$ and Borel sets $S \subseteq G$. An example of a $K$-bi-invariant measure is the $K$-orbital measure

$$
\mu_{a}=m_{K} * \delta_{a} * m_{K}
$$


where $m_{K}$ denotes the normalized Haar measure on $K$ and $\delta_{a}$ denotes the point mass measure at $a$. The $K$-orbital measure, $\mu_{a}$, is a singular probability measure which is supported on $K a K$, and is continuous (meaning nonatomic) if $a \notin N_{G}(K)$ when viewed as a measure on the symmetric space $G / K$. These measures are the extreme points of the unit ball of the space of $K$-bi-invariant, continuous measures (see [9]). Of course, if $K g K=K a K$, then $\mu_{g}=\mu_{a}$.

Ragozin proved that if $d \geq \operatorname{dim} G / K$, then $\mu_{a_{1}} * \mu_{a_{2}} * \cdots * \mu_{a_{d}}$ is absolutely continuous with respect to Haar measure on $G$ and the $d$-fold product of double cosets $K a_{1} K a_{2} \cdots K a_{d} K$ has nonempty interior if $a_{j} \notin N_{G}(K)$. For special orbital measures the number of convolution powers can be reduced to two. Here is our main result.

THEOREM 3.1. Suppose $a_{1}, a_{2} \in \exp \mathfrak{a}$ are regular elements and $\mu_{a_{1}}, \mu_{a_{2}}$ are the associated $K$-orbital measures. Then $\mu_{a_{1}} * \mu_{a_{2}}$ is absolutely continuous with respect to Haar measure on $G$ and $K a_{1} K a_{2} K$ has nonempty interior in $G$.

PROOF. For any two elements $a_{1}, a_{2} \in \exp \mathfrak{a}$, let $f_{a_{1}, a_{2}}: K^{3} \rightarrow G$ be given by

$$
f\left(k_{0}, k_{1}, k_{2}\right)=k_{0} a_{1} k_{1} a_{2} k_{2} \text {. }
$$

The proof of [9, Theorem 2.5] (an application of the implicit function theorem) shows that if the rank of $f_{a_{1}, a_{2}}$ is full, except possibly on a set of Haar measure zero, for each $a_{1}, a_{2}$ in the support of the $K$-bi-invariant measures $\mu_{1}, \mu_{2}$, then $\mu_{1} * \mu_{2}$ is absolutely continuous and $K \mathrm{~K}_{1} \mathrm{Ka}_{2} \mathrm{~K}$ has nonempty interior. However, an analyticity argument proves that if the rank of $f_{a_{1}, a_{2}}$ is full at one point, then it is full on a set whose complement has measure zero.

Thus to prove our theorem it will be enough to show that whenever $a_{1}, a_{2}$ are two regular elements in $\exp \mathfrak{a}$, then the rank $f_{a_{1}, a_{2}}$ is full at one point, and this is what we shall prove. For notational convenience we shall write $f$ for $f_{a_{1}, a_{2}}$.

The differential of $f$ at the point $\left(k_{0}, k_{1}, k_{2}\right),\left.d f\right|_{\left(k_{0}, k_{1}, k_{2}\right)}$, is the map from $\mathfrak{k}^{3}$ to $\mathfrak{g}$ given by

$$
\left.d f\right|_{\left(k_{0}, k_{1}, k_{2}\right)}\left(X_{0}, X_{1}, X_{2}\right)=-\left(X_{0}+\operatorname{Ad}\left(k_{0} a_{1}\right) X_{1}+\operatorname{Ad}\left(k_{0} a_{1} k_{1} a_{2}\right) X_{2}\right) k_{0} a_{1} k_{1} a_{2} k_{2}
$$

for $X_{i} \in k$. (This is true because of our convention of using right invariant vector fields.) Thus rank $f$ at $\left(k_{0}, k_{1}, k_{2}\right)$ is the dimension of

$$
\operatorname{span}\left\{X_{0}+\operatorname{Ad}\left(k_{0} a_{1}\right) X_{1}+\operatorname{Ad}\left(k_{0} a_{1} k_{1} a_{2}\right) X_{2} \mid X_{0}, X_{1}, X_{2} \in \mathfrak{k}\right\},
$$

which is equal to the dimension of

$$
\operatorname{span}\left\{X_{0}+\operatorname{Ad}\left(a_{1}\right) X_{1}+\operatorname{Ad}\left(a_{1} k_{1} a_{2}\right) X_{2} \mid X_{0}, X_{1}, X_{2} \in \mathfrak{k}\right\} .
$$

Hence it is enough to show that there exists a point $k_{1} \in K$ such that

$$
\mathfrak{k}+\operatorname{Ad}\left(a_{1}\right) \mathfrak{k}+\operatorname{Ad}\left(a_{1} k_{1} a_{2}\right) \mathfrak{k}=\mathfrak{g},
$$


or, equivalently,

$$
\mathfrak{k}+\operatorname{Ad}\left(a_{1}^{-1}\right) \mathfrak{k}+\operatorname{Ad}\left(k_{1} a_{2}\right) \mathfrak{k}=\mathfrak{g} .
$$

Note that Corollary 2.2 implies that $\mathfrak{k}+\operatorname{Ad}\left(a_{1}^{-1}\right) \mathfrak{k}=\mathfrak{g} \ominus \mathfrak{a}$.

As in the previous section, let $\mathcal{P}$ be the projection operator defined from $\mathfrak{g}$ onto $\mathfrak{a}$. Using this notation, it follows that to prove the theorem it suffices to show that for each $a$ regular, there exists some $k \in K$ for which $\operatorname{dim}(\mathcal{P}(\operatorname{Ad}(k a) \mathfrak{k}))=\operatorname{dim}(\mathfrak{a})$.

According to Lemma 2.4, for each positive, restricted root $\alpha$ it is possible to choose a restricted root vector $E_{\alpha} \in g_{\alpha}^{R}$ satisfying $\left[E_{\alpha}, \theta\left(E_{\alpha}\right)\right] \in i \mathfrak{a}$. Define $F_{\alpha}, F_{\alpha}^{\prime}, G_{\alpha}, G_{\alpha}^{\prime}$ as described in the previous section with this choice of $E_{\alpha}$. Set

$$
Z=\sum_{\beta \in \Sigma^{+}} F_{\beta}+F_{\beta}^{\prime}
$$

and for any real number $s$ put $k_{s}=\exp (s Z)$. Since $Z \in \mathfrak{k}, k_{s}$ belongs to the subgroup $K$.

Fix $a \in \exp \mathfrak{a}, a$ regular. For $\alpha \in \Sigma^{+}$,

$$
\begin{aligned}
\operatorname{Ad}\left(k_{s} a\right)\left(F_{\alpha}+F_{\alpha}^{\prime}\right) & =\operatorname{Ad}\left(k_{s}\right)\left(\cos \alpha(a)\left(F_{\alpha}+F_{\alpha}^{\prime}\right)-\sin \alpha(a)\left(G_{\alpha}-G_{\alpha}^{\prime}\right)\right) \\
& =\exp (a d(s Z))\left(\cos \alpha(a)\left(F_{\alpha}+F_{\alpha}^{\prime}\right)-\sin \alpha(a)\left(G_{\alpha}-G_{\alpha}^{\prime}\right)\right) \\
& =\cos \alpha(a)\left(F_{\alpha}+F_{\alpha}^{\prime}\right)-\sin \alpha(a)\left(G_{\alpha}-G_{\alpha}^{\prime}\right)+R+S,
\end{aligned}
$$

where

$$
R=s\left[Z, \cos \alpha(a)\left(F_{\alpha}+F_{\alpha}^{\prime}\right)-\sin \alpha(a)\left(G_{\alpha}-G_{\alpha}^{\prime}\right)\right]
$$

and

$$
S=\sum_{l=2}^{\infty} \frac{s^{l}}{l !}(a d Z)^{l}\left(\cos \alpha(a)\left(F_{\alpha}+F_{\alpha}^{\prime}\right)-\sin \alpha(a)\left(G_{\alpha}-G_{\alpha}^{\prime}\right)\right) .
$$

Since $Z, F_{\alpha}, F_{\alpha}^{\prime} \in \mathfrak{k}$, we have $\mathcal{P}\left[Z, F_{\alpha}+F_{\alpha}^{\prime}\right]=0$ for all $\alpha \in \Sigma$. Also, $F_{\alpha}, F_{\alpha}^{\prime}$, $G_{\alpha}, G_{\alpha}^{\prime} \in \sum_{\alpha \in \Sigma} g_{\alpha}^{R}$, hence $\mathcal{P}\left(F_{\alpha}+F_{\alpha}^{\prime}\right)=0=P\left(G_{\alpha}-G_{\alpha}^{\prime}\right)$. Therefore

$$
\mathcal{P}\left(\operatorname{Ad}\left(k_{s} a\right)\left(F_{\alpha}+F_{\alpha}^{\prime}\right)\right)=-s \sin \alpha(a) \mathcal{P}\left(\left[Z, G_{\alpha}-G_{\alpha}^{\prime}\right]+s Y_{\alpha, s}\right)
$$

where

$$
Y_{\alpha, s}=\sum_{l=2}^{\infty} \frac{s^{l-2}}{l !}(\operatorname{ad} Z)^{l}\left(G_{\alpha}-G_{\alpha}^{\prime}\right)
$$

First, consider

$$
\begin{aligned}
{\left[Z, G_{\alpha}-G_{\alpha}^{\prime}\right] } & =\left[\sum_{\beta \in \Sigma^{+}} F_{\beta}+F_{\beta}^{\prime}, G_{\alpha}-G_{\alpha}^{\prime}\right] \\
& =\left[F_{\alpha}+F_{\alpha}^{\prime}, G_{\alpha}-G_{\alpha}^{\prime}\right]+\sum_{\beta \neq \alpha}\left[F_{\beta}+F_{\beta}^{\prime}, G_{\alpha}-G_{\alpha}^{\prime}\right]
\end{aligned}
$$


If $\beta \neq \alpha$, then also $\beta \neq-\alpha$ since $\beta$ and $\alpha$ are positive, restricted roots. Hence either $\left[F_{\beta}+F_{\beta}^{\prime}, G_{\alpha}-G_{\alpha}^{\prime}\right] \in \sum_{\gamma= \pm \alpha \pm \beta} g_{\gamma}^{R}$ or none of $\pm \alpha \pm \beta$ are roots, in which case $\left[F_{\beta}+F_{\beta}^{\prime}, G_{\alpha}-G_{\alpha}^{\prime}\right]=0\left[1\right.$, Proposition 32.5]. In either case, $\mathcal{P}\left[F_{\beta}+F_{\beta}^{\prime}\right.$, $\left.G_{\alpha}-G_{\alpha}^{\prime}\right]=0$.

Combined with Lemmas 2.5 and 2.6, this observation implies that

$$
\begin{aligned}
\mathcal{P}\left(\left[Z,\left(G_{\alpha}-G_{\alpha}^{\prime}\right)\right]\right) & =\mathcal{P}\left(\left[F_{\alpha}+F_{\alpha}^{\prime}, G_{\alpha}-G_{\alpha}^{\prime}\right]\right) \\
& =\mathcal{P}\left(\left[F_{\alpha}^{\prime}, G_{\alpha}\right]-\left[F_{\alpha}, G_{\alpha}^{\prime}\right]\right) \\
& =-8 \mathcal{P} i\left[E_{\alpha}, \tau E_{\alpha}\right]=\left.c_{\alpha} H_{\alpha}\right|_{\mathfrak{a}}
\end{aligned}
$$

for some nonzero constant $c_{\alpha}$. Hence

$$
\mathcal{P}\left(\operatorname{Ad}\left(k_{s} a\right)\left(F_{\alpha}+F_{\alpha}^{\prime}\right)\right)=-s \sin \alpha(a)\left(\left.c_{\alpha} H_{\alpha}\right|_{\mathfrak{a}}+s \mathcal{P}\left(Y_{\alpha, s}\right)\right) .
$$

Thus to show that $\operatorname{dim}(\mathcal{P}(\operatorname{Ad}(k a) \mathfrak{k}))=\operatorname{dim}(\mathfrak{a})$ it is enough to prove that for suitably small $s$, the set

$$
\left\{\mathcal{P}\left(\operatorname{Ad}\left(k_{s} a\right)\left(F_{\alpha}+F_{\alpha}^{\prime}\right)\right) \mid \alpha \in \Sigma\right\}
$$

or, equivalently,

$$
\left\{\left.c_{\alpha} H_{\alpha}\right|_{\mathfrak{a}}+s \mathcal{P}\left(Y_{\alpha, s}\right) \mid \alpha \in \Sigma\right\}
$$

contains a linearly independent set of size $\operatorname{dim} \mathfrak{a} \equiv r$. To see that this is true, choose positive, restricted roots, $\alpha_{1}, \ldots, \alpha_{r}$, such that $\left\{\left.H_{\alpha_{j}}\right|_{\mathfrak{a}} \mid j=1, \ldots, r\right\}$ is a basis for $\mathfrak{a}$. We claim that the set of vectors

$$
\left\{\left.c_{\alpha_{j}} H_{\alpha_{j}}\right|_{\mathfrak{a}}+s \mathcal{P}\left(Y_{\alpha_{j}, s}\right) \mid j=1, \ldots, r\right\}
$$

is linearly independent for sufficiently small $s$.

Assume otherwise, say,

$$
\sum_{j=1}^{r} d_{j}\left(\left.c_{\alpha_{j}} H_{\alpha_{j}}\right|_{\mathfrak{a}}+s \mathcal{P}\left(Y_{\alpha_{j}, s}\right)\right)=0
$$

with not all $d_{j}=0$. Since all norms are comparable on a finite-dimensional space, there exists a positive constant $C_{0}$ such that

$$
\left\|\sum_{j=1}^{r} d_{j} c_{\alpha_{j}} H_{\alpha_{j}}|\mathfrak{a}|\right\| \geq C_{0} \sum_{j=1}^{r}\left|d_{j} c_{\alpha_{j}}\right| \geq C_{0} \min \left|c_{\alpha_{j}}\right| \sum_{j=1}^{r}\left|d_{j}\right| .
$$

For any $0<s<1$,

$$
\left\|\mathcal{P} Y_{\alpha, s}\right\| \leq\left\|Y_{\alpha, s}\right\| \leq \sum_{l=2}^{\infty} \frac{\|a d Z\|^{l} \max _{\alpha \in \Sigma} \|\left(G_{\alpha}-G_{\alpha}^{\prime} \|\right.}{l !} \equiv C_{Z}
$$


where $C_{Z}$ is independent of $\alpha$ and $s$. Hence

$$
\left\|\sum_{j=1}^{r} d_{j} s \mathcal{P}\left(Y_{\alpha_{j}, s}\right)\right\| \leq s \sum_{j=1}^{r}\left|d_{j}\right| C_{Z} .
$$

If we take $s<C_{0} \min \left|c_{\alpha_{j}}\right| / C_{Z}$ we clearly cannot satisfy (3.1) and therefore $\operatorname{dim}(\mathcal{P}(\operatorname{Ad}(k a) \mathfrak{k}))=\operatorname{dim}(\mathfrak{a})$. This completes the proof that $f$ has full rank at one point.

Corollary 3.2. Suppose $\mu_{1}, \mu_{2}$ are $K$-bi-invariant measures, compactly supported on $\bigcup_{a \in D} K a K$ where $D$ is the dense set of regular elements. Then $\mu_{1} * \mu_{2}$ is absolutely continuous.

PROOF. This can also be deduced from the same proof, as per the remarks in the first paragraph.

Corollary 3.3. Suppose $G / K$ is a compact symmetric space which admits only one positive restricted root. Then for any $a_{1}, a_{2} \notin N_{G}(K), \mu_{a_{1}} * \mu_{a_{2}}$ is absolutely continuous.

PROOF. When there is only one positive restricted root any element of exp $\mathfrak{a}$ is either in the normalizer or regular.

REMARK. Many of the rank-one symmetric spaces, including $S U(2) / S O(2)$ and $S O(p+1) / O(p)$, have only one positive restricted root. It would be interesting to know if the conclusion of the corollary holds for all rank-one spaces.

\section{Acknowledgements}

The authors are very grateful to F. Ricci for helpful conversations. The first author would also like to thank the Dept. of Pure Mathematics at the University of Waterloo for their hospitality while some of this research was done.

\section{References}

[1] D. Bump, Lie Groups, Graduate Texts in Mathematics, 225 (Springer, New York, 2004).

[2] J-L. Clerc, 'Fonctions sphériques des espaces symetriques compacts', Trans. Amer. Math. Soc. 306 (1988), 421-431.

[3] A. Dooley, J. Repka and N. Wildberger, 'Sums of adjoint orbits', Linear Multilinear Algebra 36 (1993), 79-101.

[4] C. Dunkl, 'Operators and harmonic analysis on the sphere', Trans. Amer. Math. Soc. 125 (1966), $250-263$.

[5] S. Gupta and K. E. Hare, 'Smoothness of convolution powers of orbital measures on the symmetric space $S U(n) / S O(n)$ ', Monatsh. Math. to appear.

[6] K. E. Hare, 'The size of characters of compact Lie groups', Studia Math. 129 (1998), 1-18.

[7] S. Helgason, Differential Geometry, Lie Groups and Symmetric Spaces, Pure and Applied Mathematics, 80 (Academic Press, New York, 1978). 
[8] - Groups and Geometric Analysis. Integral Geometry, Invariant Differential Operators, and Spherical Functions, Pure and Applied Mathematics, 113 (Academic Press, Inc, Orlando, FL, 1984).

[9] D. Ragozin, 'Zonal measure algebras on isotropy irreducible homogeneous spaces', J. Funct. Anal. 17 (1974), 355-376.

[10] F. Ricci and E. Stein, 'Harmonic analysis on nilpotent groups and singular integrals. II. Singular kernels supported on submanifolds', J. Funct. Anal. 78 (1988), 56-84.

[11] F. Ricci and G. Travaglini, ' $L^{p}-L^{q}$ estimates for orbital measures and Radon transform on compact Lie groups and Lie algebras', J. Funct. Anal. 129 (1995), 132-147.

SANJIV KUMAR GUPTA, Department of Mathematics and Statistics, Sultan Qaboos University, PO Box 36, Al Khodh 123, Sultanate of Oman e-mail: gupta@squ.edu.om

KATHRYN E. HARE, Department of Pure Mathematics, University of Waterloo, Waterloo, Ontario, Canada, N2L 3G1

e-mail: kehare@uwaterloo.ca 